\title{
The visitor from an ancient galaxy: A planetary companion around an old, metal-poor red horizontal branch star
}

\author{
Rainer J. Klement ${ }^{1}$, Johny Setiawan ${ }^{1}$, Thomas Henning ${ }^{1}$, \\ Hans-Walter Rix ${ }^{1}$, Boyke Rochau ${ }^{1}$, Jens Rodmann ${ }^{2}$ \\ and Tim Schulze-Hartung ${ }^{1}$ \\ ${ }^{1}$ Max-Planck-Institute for Astronomy \\ Königstuhl 17, D-69121 Heidelberg, Germany \\ email: klement@mpia.de \\ ${ }^{2}$ European Space Agency, Space Environment and Effects Section, ESTEC
}

\begin{abstract}
We report the detection of a planetary companion around HIP 13044, a metal-poor red horizontal branch star belonging to a stellar halo stream that results from the disruption of an ancient Milky Way satellite galaxy. The detection is based on radial velocity observations with FEROS at the 2.2-m MPG/ESO telescope. The periodic radial velocity variation of $P=16.2$ days can be distinguished from the periods of the stellar activity indicators. We computed a minimum planetary mass of $1.25 \mathrm{M}_{\mathrm{jup}}$ and an orbital semimajor axis of $0.116 \mathrm{AU}$ for the planet. This discovery is unique in three aspects: First, it is the first planet detection around a star with a metallicity much lower than few percent of the solar value; second, the planet host star resides in a stellar evolutionary stage that is still unexplored in the exoplanet surveys; third, the planetary system HIP 13044 most likely has an extragalactic origin in a disrupted former satellite of the Milky Way.
\end{abstract}

Keywords. galaxy: solar neighborhood, planetary systems: formation, stars: horizontal-branch, stars: individual (HIP 13044), techniques: radial velocities

\section{Introduction}

Do planets exist in external galaxies? There is no reason that they should not. However, directly observing stars in one of the Milky Way's satellites in order to obtain precise enough radial velocity or transit photometry measurements to detect planetary signals is not possible because the galaxies are too far away. $\dagger$ Although microlensing would in principal allow for extra-galactic planet detections, this phenomenon is intrinsically non-repeatable, and no microlensing planets in other galaxies have been confirmed yet (see, however, Ingrosso et al. 2009). Nevertheless, we know that certain stars in our solar neighborhood have been accreted from satallite galaxies in the past and in fact, examples of such accretion events are still observable today (e.g. Belokurov et al. 2006). Accreted stars therefore provide an opportunity to indirectly search for planets that originated in external galaxies and travelled into our Galaxy along with their host stars. In particular, stars belonging to nearby stellar halo streams are ideal targets to search for extra-galactic planets. In the inner halo, where the orbital frequencies are high, such streams are no

$\dagger$ With a distance modulus of $\sim 14.5$, the Canis Major dwarf galaxy could pose an exception, allowing at least principally for planet searches around giant stars; but it is located behind the Milky Way's disk $\left(l=240^{\circ}, b=-8^{\circ}\right)$ and therefore heavily obscured by dust and foreground light (Martin et al. 2004; Bellazzini et al. 2004). 
Table 1: Important stellar parameters of HIP 13044.

\begin{tabular}{llll}
\hline Parameter & Value & Unit & Reference \\
\hline Spectral type & $\mathrm{F} 2$ & & SIMBAD \\
$m_{V}$ & 9.94 & mag & Hipparcos \\
$T_{\text {eff }}$ & $6025 \pm 63$ & $\mathrm{~K}$ & Carney et al. $(2008 \mathrm{a}) ;$ Roederer et al. $(2010)$ \\
$R_{*}$ & $6.7 \pm 0.3$ & $\mathrm{R}_{\odot}$ & Carney et al. $(2008 \mathrm{a})$ \\
{$[\mathrm{Fe} / \mathrm{H}]$} & $-2.09 \pm 0.26$ & & $\begin{array}{l}\text { Beers } \text { et al. }(1990) ; \text { Chiba \& Beers }(2000) ; \\
\end{array}$ \\
$v \sin i$ & $8.8 \pm 0.8$ & $\mathrm{~km} \mathrm{~s}^{-1}$ & Carney et al. $(2008 \mathrm{~b}) ;$ Roederer et al. (2010) \\
& $11.7 \pm 1.0$ & $\mathrm{~km} \mathrm{~s}^{-1}$ & own measurement \\
\hline \hline
\end{tabular}

longer spatially coherent, but they still share similar chemical and dynamical properties that depend on the composition and orbit of their progenitor system and allow for identifying individual stream members (see e.g. the review by Klement 2010).

The most significant stellar halo stream in the solar neighborhood is the one discovered by Helmi et al. (1999). Its members have kinematics that clearly separate them from the bulk of other halo stars. The stream's progenitor system possibly resembled a galaxy similar to Fornax or the Sagittarius dwarf, and has been disrupted about 6-9 Gyr ago (Helmi et al. 1999; Kepley et al. 2007). The Red Horizontal Branch (RHB) star HIP 13044 is a confirmed member of the Helmi stream (Helmi et al. 1999; Re Fiorentin et al. 2005; Kepley et al. 2007; Roederer et al. 2010). Its stellar parameters, in particular the low metallicity and relatively large projected rotational velocity (Table 1), mark this star as an interesting target for a planet search.

\section{Data and Analysis}

We have obtained 36 radial velocity (RV) measurements of HIP 13044 between September 2009 and July 2010 with FEROS, a high-resolution spectrograph $(R=48,000)$ attached at the 2.2 meter Max-Planck Gesellschaft/European Southern Observatory telescope, located at the La Silla observatory in Chile (Kaufer et al. 2000). RV values have been obtained through cross-correlating the stellar spectrum with a numerical template designed for stars of the spectral type F0 and containing 550 selected spectral lines. Typical uncertainties of the RV values are $\sim 50 \mathrm{~m} \mathrm{~s}^{-1}$.

The variation of the RV between our observations at different epochs has a semiamplitude of $120 \mathrm{~ms}^{-1}$ (Fig. 2). The Generalized Lomb Scargle (GLS) periodogram (Zechmeister \& Kürster 2009) reveals a significant RV periodicity at $P=16.2$ days with a False Alarm Probability (FAP) of $5.5 \times 10^{-6}$. Additional analysis by a Bayesian algorithm (Gregory 2005) confirms this period. To find out whether stellar activity (moving/rotating surface inhomogeneities or stellar pulsations) is responsible for producing the observed RV variation, we investigated different stellar activity indicators.

We analyzed the variation of two independent spectroscopic activity indicators: the bisector velocity spans (Hatzes 1996) of all 550 lines and the equivalent width (EW) of the infrared CaII line at $\lambda=849.8 \mathrm{~nm}$. The GLS periodogram analyses for both the BVS and the Ca II line EW variations revealed significant peaks at similar periodicities $\left(P_{\mathrm{BVS}}=5.02 \mathrm{~d}, \mathrm{FAP}_{\mathrm{BVS}}=1.4 \times 10^{-5}\right.$ and $\left.P_{\mathrm{EW}}=6.31 \mathrm{~d}, \mathrm{FAP}_{\mathrm{EW}}=4.4 \times 10^{-6}\right) . \mathrm{We}$ attribute these variations in the spectral line indicators to rotational modulation caused by star spots or large granulation cells. We point out that we have found no hints for a period around 16 days in the BSV and EW variations. 
Photometric observations of HIP 13044 have been made by Super-WASP. There exist 3620 high-precision measurements of HIP 13044 in the public Super-WASP archive. After removing 10 data points with high error bars, the Lomb-Scargle periodogram shows that there is no signal for $\mathrm{a} \sim 16 \mathrm{~d}$ period (Fig. 1b). Instead, there are several marginally significant peaks between a few hours and a few days $(\mathrm{FAP}<1 \%$ ), with the ones at $1.4 \mathrm{~d}$ and $3.5 \mathrm{~d}$ beeing the most significant. These two peaks are, however, most likely harmonic to each other: $1.4^{-1}+3.5^{-1}=1$. It is expected that HIP 13044 oscillates only at pulsationally unstable overtones of high order (Xiong et al. 1998). Observations of one RHB star in the metal-poor globular cluster NGC 6397 (Stello \& Gilliland 2009) as well as theoretical predictions (Xiong et al. 1998) set these periods in the range of a few hours to a day or so. We caution, however, that no clear theoretical predictions for a star with parameters similar to HIP 13044 exist, and it could be possible that some high-order oscillations are able to explain the 1.4 or 3.5 day signal. More important, however, is that we found no signal of a period around $16.2 \mathrm{~d}$ in the photometric data.

\section{Discussion and Conclusions}

From the lack of $\mathrm{a} \sim 16 \mathrm{~d}$ period in both photometric and spectroscopic activity indicators, we conclude that a (sub-stellar) companion remains the most likely hypothesis for the observed $16.2 \mathrm{~d}$ RV variation. Table 2 lists the orbital solution for HIP $13044 \mathrm{~b}$ that we have computed from the stellar reflex motion. While the semi-major axis is not unusual for a giant extra-solar planet, the non-circular orbit $(e=0.25)$ for such a close-in companion is. One has to keep in mind, however, that the star's red giant branch (RGB) phase, which preceeds the RHB phase, probably has changed the orbital properties of the planet. From the lack of close-in $(\lesssim 0.5$ AU, e.g. Sato et al. 2008) giant planets around RGB stars, it seems plausible that such planets, if they existed, have been engulfed during the expansion of the stellar atmosphere (although other explanations exist, e.g. Currie 2009). Such planet-swallowing would spin up the star and increase the mass loss (Soker 1998; Carlberg et al. 2009), and indeed enhanced rotation among RGB and RHB stars has been found (Carney et al. 2003, 2008a). Interestingly, HIP 13044 is such

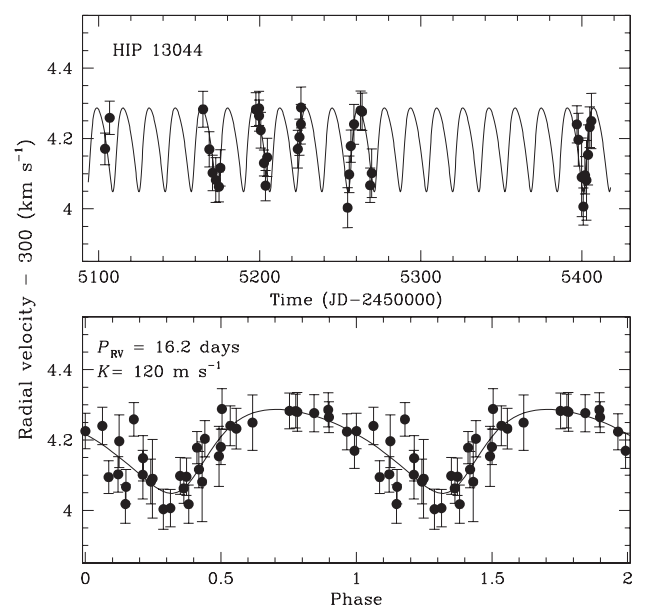

(a)

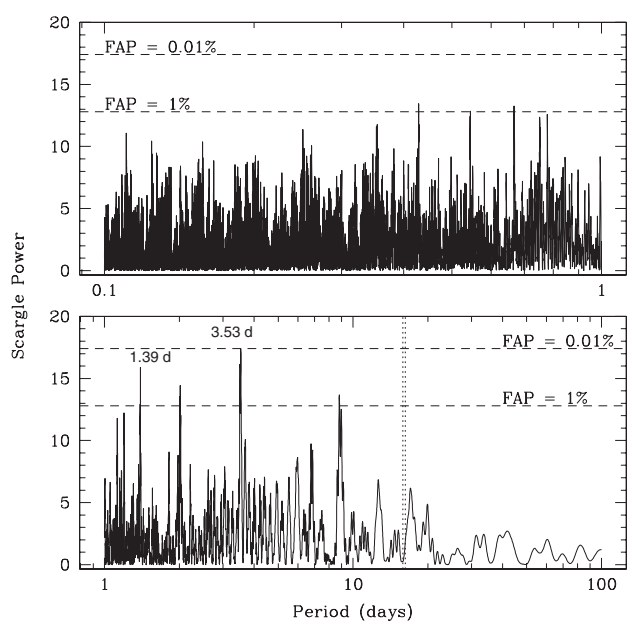

(b)

Figure 1: (a) RV variation of HIP 13044. (b) LS-periodogram of the Super-WASP data. 
a fast rotator $\dagger$ too, so possibly HIP 13044 b was part of a multiple planetary system of which some planets have not survived the RGB phase. The survival of HIP $13044 \mathrm{~b}$ during that phase is theoretically possible under certain circumstances (Soker 1998). It is also possible that the planet's orbit decayed through tidal interaction with the stellar envelope. However, a prerequisite to survival is then that the mass loss of the star stops before the planet would have been evaporated or accreted. Assuming asymmetric mass loss, velocity kicks could have increased the eccentricity of HIP 13044 b to its current, somewhat high value (Heyl 2007). The same could be achieved by interaction with a third body in the system. The formation of the planet(s) around such a metal-poor star is possible via gravitational instability. Even if we assume that the star's metallicty was higher in the past, the fact that it belongs to the chemically homogenous Helmi stream $(\langle[\mathrm{Fe} / \mathrm{H}]\rangle=-1.8 \pm 0.4$ for 33 stream members from Kepley et al. 2007; Klement et al. 2009) still implies the lowest metallicty of a planet host star found to date. The reason why most planet searches around metal-poor stars failed could be twofold: first, most planets around such stars should reside at large orbital distances (e.g. Marchi 2007; Santos et al. 2010); we could have been lucky that the RGB phase lead to an orbital decay of HIP 13044 b. Second, most planet searches tried to observed a large sample simultaneously (e.g. Sozzetti et al. 2009), in this way eventually not obtaining enough data points for a significant RV signal.

We can be pretty sure that the planet was not captured from another star inside the Milky Way, because the time on which stellar encounters play a role exceeds the Hubble time for galaxies like the Milky Way (Binney \& Tremaine 2008). The stream membership of HIP 13044 therefore implies an extra-Galactic origin of HIP 13044 b.

\begin{tabular}{cll} 
Table 2: Orbital parameters of HIP 13044 & $\mathrm{~b}$ \\
\hline \hline$P$ & $16.2 \pm 0.3$ & days \\
$K_{1}$ & $119.9 \pm 9.8$ & $\mathrm{~m} \mathrm{~s}^{-1}$ \\
$e$ & $0.25 \pm 0.05$ & \\
$\omega$ & $219.8 \pm 1.8$ & $\mathrm{deg}$ \\
$J D_{0}-2450000$ & $5109.78 \pm 0.02$ & days \\
$\chi^{2}$ & 32.35 & $\mathrm{~m} \mathrm{~s}^{-1}$ \\
$\mathrm{rms}$ & 50.86 & $\mathrm{~m} \mathrm{~s}^{-1}$ \\
$m_{2} \sin i$ & $1.25 \pm 0.05$ & $\mathrm{M}_{\text {Jup }}$ \\
$a$ & $0.116 \pm 0.01$ & $\mathrm{AU}$ \\
\hline
\end{tabular}

\section{References}

Beers, T. C., Kage, J. A., Preston, G. W., \& Shectman, S. A. 1990, AJ, 100, 849

Bellazzini, M., Ibata, R., Monaco, L., Martin, N., Irwin, M. J., \& Lewis, G. F. 2004, MNRAS, 354,1263

Belokurov, V., Zucker, D. B., Evans, N. W., Gilmore, G., Vidrih, S., et al. 2006, ApJL, 642, L137

Binney, J. \& Tremaine, S. 2008, Galactic Dynamics (Princeton University Press)

Carlberg, J. K., Majewski, S. R., \& Arras, P. 2009, ApJ, 700, 832

Carney, B. W., Latham, D. W., Stefanik, R. P., Laird, J. B., \& Morse, J. A. 2003, ApJ, 125, 293

Carney, B. W., Latham, D. W., Stefanik, R. P., \& Laird, J. B. 2008, AJ, 135, 196

Carney, B. W., Gray, D. F., Yong, D., Latham, D. W., Manset, N., Zelman, R., \& Laird, J. B. $2008, A J, 135,892$

Chiba, M. \& Beers, T. C. 2000, AJ, 119, 2843

$\dagger v_{\text {rot }} \approx 60 \mathrm{~km} \mathrm{~s}^{-1}$, following from $P_{\text {rot }} / \sin i=2 \pi R_{*} /\left(v_{\text {rot }} \sin i\right)$, with $R_{*}$ from Table 1 and $P_{\text {rot }} \approx 5.5 \mathrm{~d}$ from the BVS and EW variations. 
Currie, T. 2009, ApJL, 694, L171

Gregory, P. C. 2005, Bayesian Logical Data Analysis for the Physical Sciences: A Comparative Approach with 'Mathematica' Support (Cambridge University Press)

Hatzes, A. P. 1996, PASP, 108, 839

Helmi, A., White, S. D. M., de Zeeuw, P. T., \& Zhao, H. 1999, Nature, 402, 53

Heyl, J. 2007, MNRAS, 382, 915

Ingrosso, G., Calchi Novati, S., De Paolis, F., Jetzer, Ph., Nucita, A. A., \& Zakharov, A. F. 2009, MNRAS, 399, 219

Kaufer, A., Stahl, O., Tubbesing, S., Norregaard, P., Avila, G., et al. 2000, in: M. Iye \& A. F. Moorwood (eds.), Proc. SPIE, 4008, 459

Kepley, A. A., Morrison, H. L., Helmi, A., Kinman, T. D., Van Duyne, J., et al. 2007, AJ, 134, 1579

Klement, R., Rix, H.-W., Flynn, C., Fuchs, B., Beers, T. C., et al. 2009, ApJ, 698, 865

Klement, R. J. 2010, $A \& A R, 18,567$

Marchi, S. 2007, ApJ, 666, 475

Martin, N. F., Ibata, R. A., Bellazzini, M., Irwin, M. J., Lewis, G. F., \& Dehnen, W. 2004, MNRAS, 348, 12

Re Fiorentin, P., Helmi, A., Lattanzi, M. G., \& Spagna, A. 2005, A\& A, 439, 551

Roederer, I. U., Sneden, C., Thompson, I. B., Preston, G. W., \& Shectman, S. A. 2010, ApJ, 711,573

Santos, N. C., Mayor, M., Benz, W., Bouchy, F., Figueira, P., et al. 2010, Aש̇A, 512, A47

Sato, B., Izumiura, H., Toyota, E., et al. 2008, PASJ, 60, 539

Soker, N. 1998, AJ, 116, 1308

Sozzetti, A., Torres, G., Latham, D. W., et al. 2009, ApJ, 697, 544

Stello, D. \& Gilliland, R. L. 2009, AJ, 700, 949

Xiong, D. R., Cheng, Q. L., \& Deng, L. 1998, ApJ, 500, 449

Zechmeister, M. \& Kürster, M. 2009, A\& $A$, 496, 577 